\title{
Pagdalumat sa kasanayang komunikatibo sa panahon ng bagong kadawyan
}

Especial, Mary Ann

Sorsogon State University, Philippines (Delacruzmean05@gmail.com)

Melitante, Mary Anne

Sorsogon State University, Philippines (maryanne.melitante@deped.gov.ph)

Janer, Susan

Sorsogon State University, Philippines (sihjaner@sorsu.edu.ph)

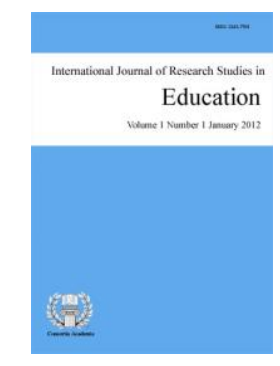

ISSN: $2243-7703$ Online ISSN: 2243-7711

OPEN ACCESS

\section{Abstract}

This study aimed to analyze the communicative skills in the new normal setting. QuantitativeQualitative research method was used in the course of completing the study. To determine the specific participants, purposive random sampling was utilized. Students' profile within the age bracket of 19-22 years old, the participants comprise of female students taking BSIS (Bachelor of Science Information System) course and are members of the youth organization SK (Sangguniang Kabataan). Accordingly, these students barely attended seminars and/or student training programs. This research used a survey- questionnaire and interview instrument in gathering the needed data. From the results accumulated, it was identified that there was a low level of communicative skills of the students in terms of strategies, independent learning skills, and materials in communication methods. Further, most problems encountered by these students were plotted around low self-confidence and lack of skills in interpersonal communication. In this study, the researchers recommended active involvement of students in community and outreach programs, community contests focused on improving communicative skills and a harness of support programs from school administration and community leaders. These helped to enhance the skills of students to not only in personal growth in communication but as much as in other various learning aspects.

Keywords: English translation skill, Filipino language, new normal, communicative, analyzing 


\section{Pagdalumat sa kasanayang komunikatibo sa panahon ng bagong kadawyan}

\section{Introduksyon}

Taong 2019 nang magsimula ang paglaganap ng COVID-19 sa Pilipinas, ito rin ang panahon na nagkaroon ng mga pagbabago, pagbabago sa panuntunan pangkalusugan at limitadong pakikipag-ugnayan. Ayon kay Bonabon (2020), sa panahong ito ng kawalang-katiyakan, dapat lamang busugin ang taumbayan, hindi lamang ng ipinamamahaging relief goods, kundi pati na rin sa mga tumpak at tiyak na impormasyon hinggil sa pagsugpo at paglaban sa COVID19. Komunikasyon ang nagbibigay buhay sa karunungan ng bawat tao. Nagpapabatid ng kaalaman, damdamin, kaisipan at maging kasaysayan ng sangkatauhan. Ang pagbasa, pagsulat at pagsasalita ay mga gawaing nagbubuklod na tatangi lamang sa kakayahan ng tao. Sa gitna ng mga pasulpot-sulpot na problemang hatid ng new normal sa mga paaralan, institusyon at pamayanan, patuloy pa ring kinakaharap ng mga mag-aaral ang mga epektong dulot nito sa kanilang edukasyon.

Ayon kay Hartley (1996), “Ang komunikasyon ay di lamang kabuuang pahayag ng kaalaman at damdamin, bagkus ito ay isa ring batayang prosesong panlipunan. Pangunahing pangangailangan ito sa pag-unlad ng indibidwal, sa patuloy na pagbuo at pananatili ng isang pangkat o lipi sa isang tiyak na lugar o lipunan."Ayon naman kay Sauco (1990), kung nais makamit ang kaangkupang pangkaisipan, pangkabuhayan, panlipunan, pangkaugalian at pang-ispiritwal, kailangan matutong linawin ang ideya at kaisipang ipaabot sa iba, gayundin, matutong umunawa at sumuri sa kahulugan at kahalagahan ng sinasabi ng kanyang kapwa.Maaari ang kasanayang pagsasalita ang pundasyon sa pakikipagtalastasan ngunit ating ding tandan na ang anumang wika o salita ay mabibigyan lamang ng kahulugan kung malinaw at komprehensibo sa pagbabahagi ng ating mga ideya.

Ang kakayahang pakikipagkomunikasyon maging pang-indibidwal o pangmadla ay mayroong mahalagang gampanin sa isang mag-aaral, napapaulad ang kaalamang gramatika, ponolohiya sa iba't ibang tunog at angkop na pagbigkas, maging sa angkop na pagbigkas ng iba’t ibang antas ng wika na siyang magagamit na kasanayan bilang aplikasyon sa kapaligiran, lipunan, pakikipag-ugnayan at pangsariling pakikipagtalastasan. Ayon sa pag-aaral nina Burney at Wrange (1998), ang pagsasalita ay komunikasyon ng mga ideya at damdamin sa pamamagitan ng mga simbolong nakikita at naririnig mula sa tagapagsalita. Publiko o pribado man, orihinal o salin, ito'y komunikasyong pasalita na naglalaman ng mga kaisipan at ideya na nagtataglay ng mga simbolo-tunog, salita, kilos at iba pa. Sinuportahan ito ni Patanao (1996), sa pagkilos na pahayag maraming daluyan (channel) ng komunikasyon na maaaring magpahiwatig sa pamamagitan ng mata, galaw o kumpas ng kamay, pangangatal ng buong katawan at pagtitiim ng mga bagang. Subalit ang lahat ng mga ipinahihiwatig sa ganitong pahayag ay di madalig makuha. Kinakailangan parin ng sensitibong pakiramdam ng pagpapahayag, upang matamo ang kaugnay na mensahe.

Sa pag-aaral ng sining ng komunikasyon ay dapat bigyan-diin ang pag-unawa sa kahulugan sa tulong ng mga makrong kasanayan. Kaya ang isang mag-aaral ay dapat magkaroon ng kasanayang pangkomunikasyon, ang paggamit ng angkop na salita sa kausap, sa lugar, sa panahon at mga pangyayari (Villamin, 1990). Ang komunikasyon bilang paglinang ng ugnayan at relasyon ay nagsisismula sa pakikipag kaibigan. Napagtitibay ng komunikasyon sa pamamagitan ng isang pagkakaisa ng komunidad o bansa. Dahil din sa komunikasyon mas madali ang pagpapalitan ng produkto ng mga bansa ito man ay pag-import or pag-export ng produkto. Ang komunikasyon ang nagsisilbing tulay ng impormasyon at maging sa paglalaganap ng kultura at paniniwala ng mga tao.

Sa kasalukuyang panahon, nagbigay ng panibagong pagsubok at oportunidad sa larangan ng edukasyon ang pag-usbong ng teknolohiya. Hudyat lamang ng pagyakap sa pagbabago sa mga estudyante sa kanilang pag-aaral ang paggamit ng makabagong teknolohiya sapagkat bahagi na ito ng kanilang karanasan at lipunan ginagalawan. Hindi na bago sa mga kabataan ang paggamit ng makabagong teknolohiya dahil ito ang kanilang nasilayan sa 
kanilang henerasyon. Nagbibigay ng kontribusyon para sa mga estudyante at sa mga guro sa larangan ng akademiko at makabuluhang pagkatuto ang kasanayan lalo na sa kanilang pag-aaral. Sa pahayag nina Johnson, et al. (2010) sa kanyang paniniwala na, "schools need to adapt to current student needs and identify new learning models that engage younger generations". Ang pagtanggap ng pagbabago ng institusyon ay isang proseso upang maging mahusay, organisado, taguyod at susuporta sa pagkatuto. Sa pagpapaunlad na ito ay inaasahang aangat ang ibubunga ng mga estudyante sa pagkatuto sa paaralan. Malaking tulong sa mga estudyante ang paggamit ng teknolohiya sa pag-aaral, mapadali nito ang pagkatuto at sa madaliang panahon.

Pinatunayan nina Zulueta at Guimbatan (2002) na dapat iangkop ng mga guro ang mga istratehiya sa pagtuturo upang umakma sa pangangailangan ng mga estudyante. Walang nag-iisang tamang paraan na magtuturo sa partikular na grupo ng mga estudyante sa klase. Sa punto ng kanilang pahayag, nagpapahiwatig ito na hindi dapat na alisin ng guro ang kanyang responsibilidad sapagkat mahalaga ang papel niya sa pag-iisip ng mga estratehiyang magiging dinamiko at epektibo ang magiging kahihinatnan ng kanyang pagtuturo. Isa sa makrong kasanayan ang pagsasalita na naghahatid ng mensahe sa paraang berbal sa aralin ayon sa resulta ng pag-aaral nina Saul, A. J. G. et al. (2020) na Manipestasyon ng Ika-21 Siglong Kasanayan at Mungkahing Gawaing Makalilinang ng Kasanayan sa Kurikulum ng Filipino ng Ikasampung Baitang.

Sa pag-aaral ni Mandado (2020) na sa pagtuturo ng Filipino ay hinanapan ng mga pamaraan upang matugunan ang mga pangangilangan ng ika-21 siglong mag-aaral. Sadyang mahalaga ang pagsabay sa pagusbong ng modernong panahon para makasabay sa indak at interes ng kabataan. Kaya, sa pag-aaral na ito ay mahalagang mabigyang-lunas ang suliraning kakayahang komunikatibo sa pasalita na isang nakawiwili at makabuluhang paraan. Batid ng mananaliksik ang maraming paglalapat sa mga pagtuturo ng iba't ibang larangan tulad ng pagbibigay ng pag-unawa sa paksa, pagmomonitor na mga estudyante sa mga gawain na siya namang kaagad nabibigyan ng kaukulang tugon para sa pagwawasto ng mga kamalian, at pagpapabuti sa susuod na talakayan, at paghahanda para sa susunod na talakayan.Isang hamon para sa mga mag-aaral sa kolehiyo ay ang pagpapaunlad sa kasanayang komunikatibo, higit na inaasahan ang malawak na kasanayan sa kanilang antas ngunit sa paano paraan o ano ang estratihiya ang maaring mailapat sa panahon ng pandemya. Ito ang naging hudyat sa mananaliksik na bigyang halaga ang pagiging progresibong pagtuturo sa Filipino sa kasanayang pasalita sa mga estudyante gamit ang mga pamamaraan at kagamitan sa makabagong teknolohiya.

\subsection{Layunin ng pag-aaral}

Pangunahing layunin ng pag-aaral na ito na kumalap ng impormasyong kinakailangan para tuklasin ang antas ng kasanayan komunikatibo ng mga mag-aaral sa larangan ng estratihiya sa pakikipagtalastasan, kasanayang pansarili, kasangkapan sa pakikipagtalastasan, at pamamaraan. Maging ang mga suliraning nakakahadlang sa paglinang ng kasanayan ng mga mag-aaral at makapaglatag ng mungkahing gawain ang makatulong sa resulta ng pag-aaral.

\section{Materyales at methodolohiya}

Natiyak sa pag-aaral na ito ang kasanayang komunikatibo ng mga mag-aaral sa panahon ng kadawyan. Sa pangangalap ng datos sa pag-aaral, iba't ibang paraan ang ginamit sa pangangalap ng mga datos hinggil sa pag-aaral. Kwantitatibong-kwalitatibong pamamaraan ng pag-aaral ang ginamit. At ang pagsasagawa ng sarbey gamit ang talatanungan para sa 25 na mag-aaral.Sinuri ang mga tugon ng mga estudyante batay sa saloobin mula sa ginawang talatanungan ng mananaliksik.

\subsection{Kalahokng Pag-aaral}

Ang mga mag-aaral sa Sorsogon State University-Bulan Campus ang siyang tumugon sa inihandang talatanungan upang makilala at masuri ang kanilang mga saloobin sa kasanayang komunikatibo. Purposive sampling ang ginamit ng mananaliksik sa pagtukoy ng mga kalahok ng pag-aaral. Ang profayl ng mga kalahok sap ag-aaral, na may edad na 19-22, karamihan ay mga kababaihan, at may kursong BSIS (Bachelor of Science 
Especial, M. A., Melitante, M. A., \& Janer, S.

Information System), nabibilang sa student organization na SK (Sangguniang Kabataan) at wala gaanong dinaluhan na mga palihan o student training ang mga mag-aaral.

\subsection{Instrumento ng Pananaliksik}

Ang mananaliksik ay gumamit ng balidong talatanungan upang makalap ang mga mahahalagang datos na kinakailangan sa pag-aaral. Naglalaman ang talatanungan ng profayl ng mga mag-aaral, indicator na pahayag na may layuning alamin ang antas ng kasanayan komunikatibo ng mga mag-aaral sa larangan ng estratihiya sa pakikipagtalastasan, kasanayang pansarili, kasangkapan sa pakikipagtalastasan at pamamaraan. Ang interpretasyon ng bawat antas na paglalarawan ay ginamit ang Nominal na sukatan. Ang tseklist ay may tatlong (3) pagpipilian na may leyendang: 3- Mataas ang kasanayan 2 -bahagyang mababang kasanayan 1) - mababang kasanayan. Sa pagtukoy ng suliranin kinakaharap at mungkahing gawain na makatutulong sa kasanayan ay gumamit ang mananaliksik ng pagranggo mula sa pinakaraniwang sagot ng mga mag-aaral.

\section{Paglalahad at pagsusuri ng mga datos}

Inilahad sa bahaging ito ang interprestasyon sa mga nakalap na datos na makapagbigay ng kasagutan sa mga suliraning inilahad ang mga nakuhang sagot batay sa resulta ng pag-aaral na ito.

\section{Talahanayan 1}

Antas ng kasanayan komunikatibo ng mga mag-aaral

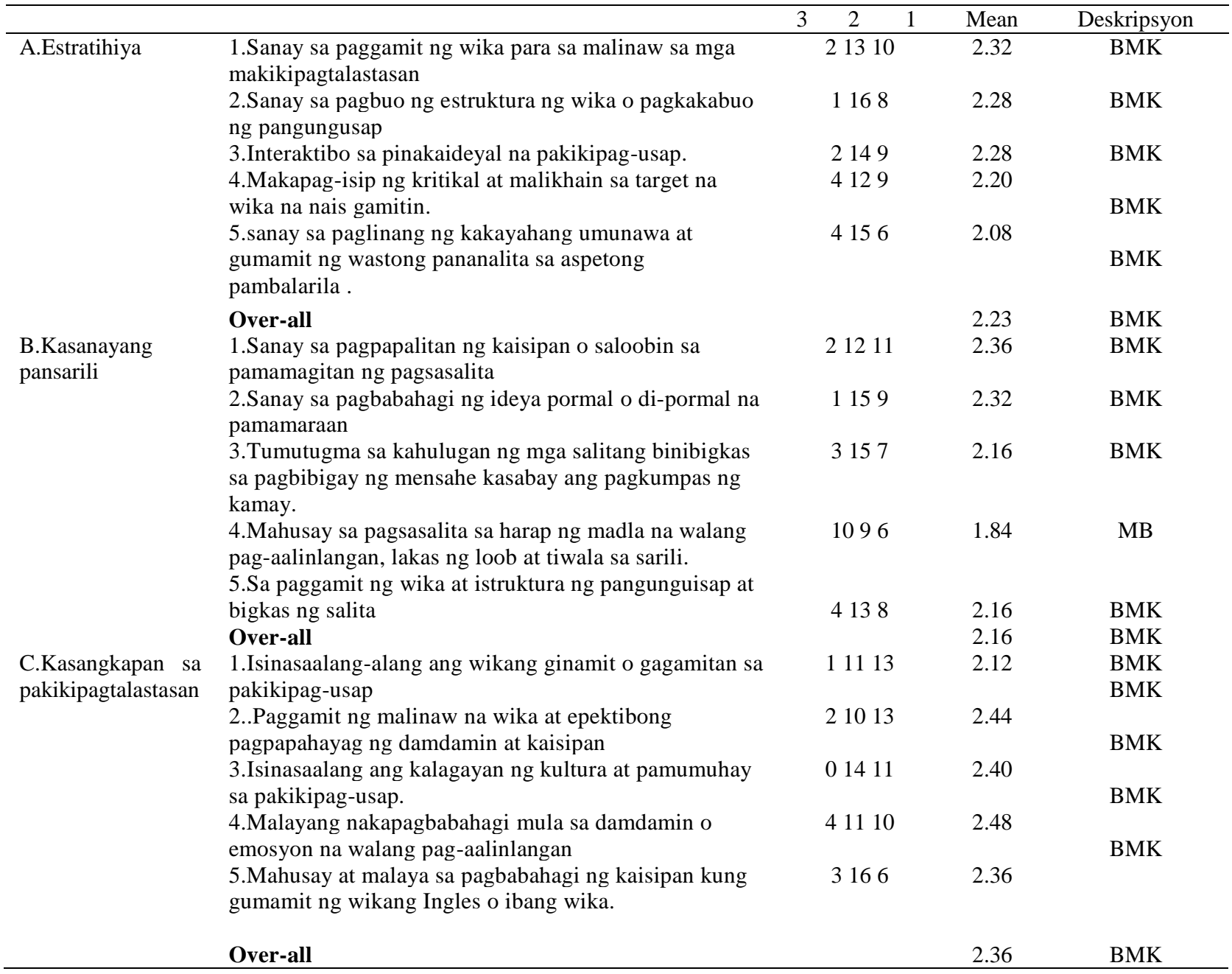


Pagdalumat sa kasanayang komunikatibo sa panahon ng bagong kadawyan

\begin{tabular}{|c|c|c|c|c|}
\hline \multirow[t]{5}{*}{$\begin{array}{l}\text { D.Pamamaraan sa } \\
\text { pakikipagtalastasan }\end{array}$} & $\begin{array}{l}\text { 1.Pagkikipag-usap gamit ang chat, messeges o email } \\
\text { (text messages) }\end{array}$ & 3166 & 2.32 & BMK \\
\hline & $\begin{array}{l}\text { 2.Pakikipag-usap gamit ang cellphone (pagtawag), } \\
\text { video call, zoom, google meet (call messages) }\end{array}$ & 21013 & 2.28 & BMK \\
\hline & $\begin{array}{l}\text { 3.Pakikipagdaupang palad na kasama o kaharap sa } \\
\text { kausap (face to face) }\end{array}$ & 11311 & 2.28 & BMK \\
\hline & $\begin{array}{l}\text { 4. Nakapagbabahagi ng ideya sa pamamagitan ng pasulat } \\
\text { o pasalaysay gamit ang papel o pasulat. }\end{array}$ & 11113 & 2.20 & BMK \\
\hline & $\begin{array}{l}\text { 5.Nakakapagbahagi ng ideya, saloobin at damdamin sa } \\
\text { pamamagitan ng mga post sa social media(FB, } \\
\text { Instagram,Twitter at iba pa.) }\end{array}$ & 31012 & 2.08 & BMK \\
\hline
\end{tabular}

\section{Grand Mean}

Over-all

2.23 2.245

Bahagyang Mababang Kasanayan

Note: 2.50-3.00 Mataas na kasanayan (3), 1.50-2.49 Bahagyang Mababang kasanayan (2), 1.0-1.49-Mababang kasanayan (1).

Makikita sa talahanayan 1 na 2.23 ang over all weighted mean sa estratihiya sa pakikipagtalastasan ng mga mag-aaral na nagsasaad na bahagyang mababang kasanayan (BMK). Ayon kay Sauco (1990), kung nais makamit ang kaangkupang pangkaisipan, pangkabuhayan, panlipunan, pangkaugalian at pang-ispiritwal, kailangan matutong linawin ang ideya at kaisipang ipaabot sa iba, gayundin, matutong umunawa at sumuri sa kahulugan at kahalagahan ng sinasabi ng kanyang kapwa.Maaari ang kasanayang pagsasalita ang pundasyon sa pakikipagtalastasan ngunit ating ding tandan na ang anumang wika o salita ay mabibigyan lamang ng kahulugan kung malinaw at komprehensibo sa pagbabahagi ng ating mga ideya.

Sa kasanayang pansarili ng mga mag-aaral lumabas na 2.16 over all weighted mean na nagsasaad na bahagyang mababang kasanayan (BMK), sa pag-aaral ng sining ng komunikasyon ay dapat bigyan-diin ang pag-unawa sa kahulugan sa tulong ng mga makrong kasanayan. Kaya ang isang mag-aaral ay dapat magkaroon ng kasanayang pangkomunikasyon, ang paggamit ng angkop na salita sa kausap, sa lugar, sa panahon at mga pangyayari (Villamin, 1990). Maaring makaapekto ang kawalan ng kasanayan sa pagsasalita sa harap ng madla, kawalan ng tiwala sa sarili. Ayon kay Hartley (1996), “Ang komunikasyon ay di lamang kabuuang pahayag ng kaalaman at damdamin, bagkus ito ay isa ring batayang prosesong panlipunan pangunahing pangangailangan ito sa pag-unlad ng indibidwal, sa patuloy na pagbuo at pananatili ng isang pangkat o lipi sa isang tiyak na lugar o lipunan."

Makikita naman sa kasangkapan sa pakikipagtalastasan ng mga mag-aaral na 2.36 na over all weighted mean na nagsasaad na bahagyang mababang kasanayan ang naipapakita. Sinuportahan ito ni Patanao (1996), sa pagkilos na pahayag maraming daluyan (channel) ng komunikasyon na maaaring magpahiwatig sa pamamagitan ng mata, galaw o kumpas ng kamay, pangangatal ng buong katawan at pagtitiim ng mga bagang. Subalit ang lahat ng mga ipinahihiwatig sa ganitong pahayag ay di madalig makuha. Kinakailangan parin ng sensitibong pakiramdam ng pagpapahayag, upang matamo ang kaugnay na mensahe.

Sa pamamaraan sa pakikipagtalastasan naman makikita na ang over all weighted mean na 2.23 nagsasaad na bahagyang mababang kasanayan. Sa pag-aaral ni Mandado (2020) na sa pagtuturo ng Filipino ay hinanapan ng mga pamaraan upang matugunan ang mga pangangilangan ng ika-21 siglong mag-aaral. Sadyang mahalaga ang pagsabay sa pag-usbong ng modernong panahon para makasabay sa indak at interes ng kabataan. Dagdag pa sa pag-aaral nina Dannah Boyd (2014), ginagamit ang sosyal medya upang makalikha ng liham o mensahe upang makagawa ng isang komunikasyon, kadalasang ginagamit ay facebook, dahil madaling gamitin. Sa panahon ngayon, malawak na ang kaalaman ng mga mag-aaral sa paggamit ng teknolohiya, kasabay ng pagbabago ng teknolohiya ang wika na may kaugnayan pakikipagtalastasan ng mga tao, umuunlad o nagbabago na maaaring maging daan upang makabahagi, makipag-unayan sa kanyang kapwa.

Sa kabuuang resulta ng pag-aaral sa antas ng kasanayang komunikatibo ng mga mag-aaral lumalabas na mayroong weighted mean na 2.245 nagsasaad na bahagyang mababang kasanayan, Ang kakayahang 
Especial, M. A., Melitante, M. A., \& Janer, S.

pakikipagkomunikasyon maging pang-indibidwal o pangmadla ay mayroong mahalagang gampanin sa isang mag-aaral, napapaulad ang kaalamang gramatika, ponolohiya sa iba't ibang tunog at angkop na pagbigkas, maging sa angkop na pagbigkas ng iba't ibang antas ng wika na siyang magagamit na kasanayan bilang aplikasyon sa kapaligiran, lipunan, pakikipag-ugnayan at pangsariling pakikipagtalastasan.

\subsection{Ano ang mga suliraning nakakahadlang sa paglinang ng kasanayan sa komunikasyon ng isang mag-aaral}

Nakapaloob sa bahaging ito ang suliraning kinahaharap ng mga mag-aaral.Narito ang ilan sa nagging pahayag ng kalahok.

$>$ Kalahok 1: "kawalan ng self-confidence"

> Kalahok 2: "ang suliraning nakahahadlang sa paglinang ng mga kasanayan sa kumunikasyon ng mga mag-aaral,ay kulang sa kasanayan dahil hindi ito naituturo bilang asignatura sa paaralan"

$>$ Kalahok 3: "Mga pag aalinlangan ng isip at distraksyon"

> Kalahok 4: "Bukod sa nahihiya, may pagkakataon na ilap sa tao o kaya nama'y natatakot magbitaw ng salita kung ma mis- interpret ng kausap at ayaw mahusgahan ayun sa way ng kanilang pagsasalita o kung sa wikang ingles naman, natatakot mahusgahan lalo na kung hindi bihasa."

> Kalahok 5: "Ilan sa mga suliraning nakakahadlang sa paglinang ng kasanayan sa komunikasyon ng mga mag-aaral ay ang kakulungan sa pakikipagtalastasan at kagamitang TEKNOLOHIYA na mas nagpapatibay sa kasanayan ng mga mag-aaral pagdating sa larangan ng komunikasyon.”

Sa aspetong pakikipagtalastasan o komunikatibo ay maaari natin kakitaan ng maraming factor kung bakit nagiging mahina o nawawalan ng kasanayan sa pakikipag- usap ang mga mag-aaral lalo na ang lahat ang kinahaharap ang pandemya. Sa isang pananaliksik na may pamagat na "Language as medium of communication" ang wika ay may importenteng papel maging sa paraang pagsulat o pananalita, bagamat hindi nagbanggit ng tiyak na wika, katulad rin ito ng konsepto at maging ang resulta ng pananaliksik na ang wikang naiintindihan ng nakakaramihan ay pinakamainam na paraan upang mas maintindihan ang impormasyon o nais ipahiwatig ng nagpapadala ng mensahe. Nabanggit sa resulta ng pananaliksik na may pamagat na "The significance of Language as a tool of Communication", ang pangkaraniwang lengwahe ang pinakaimportante sa isang pamayanan.Ang pag gamit ng iisang wika na naiintindihan ng karamihan ay mas epektibo sa paghahatid ng mensahe.

Ayon kina Hilario Et al. (n.d) nagiging susi ang wika upang magkaroon ng isang matibay at naiintindihang pakikipagtalastasan nagsilbing instrumento ito upang bigyang daan o tulay sa pagpapalitan ng saloobin at mga ideya ang mga tao. Nagiging instrumento ang komunikasyon kapag ito ay maayos na naipahayag ang saloobin at ideya na maaring maging resulta ng kasunduan at pagkakaintindihan. Ayon pa rin kina Hilario et al. (n.d) ang komunikasyon ay makakakatulong upang mapagbuklod ng mga tao, magpahayag ng damdamin, mag pahayag ng pagiging malikhain, mayaman sa talasalitaan at makapanghikayat. Isa rin mahalagang aspeto na ang mga mag-aaral ay bukas at may kamalayan sa suliranin nagiging hadlang sa pakikipagtalastasan. Indikasyon lamang ito na ang kamalayan sa suliranin ang isang positibong pagbubukas ng kagustuhan na matugunan ang kanilang pangangailangan.

\section{Kongklusyon at rekomendasyon sa pag-aaral}

Batay sa mga natuklasan nabuo ang mga sumusunod na kongklusyon:Ang antas ng kasanayan komunikatibo ng mga mag-aaral sa larangan ng estratihiya sa pakikipagtalastasan, kasanayang pansarili, kasangkapan sa pakikipagtalastasan,at pamamaraan lumabas na bahagyag mababa ang kasanayan ng mga mag-aaral.Ang mga suliraning nakakahadlang sa paglinang ng kasanayan ng mga mag-aaral, karaniwan ang kawalan ng tiwala sa sarili at kawalan ng kakayahan na makapagbahagi sa ibang tao. 
Sa kabuuan, isinasalamin ng pananaliksik na ito ang tunay na kalagayan ng mga mag-aaral hinggil sa kasanayang komunikatibo, kaya naman inirerekomenda ang mga sumusunod upang mas lalaong mapabisa ang kasanayan ng mga mag-aaral:

$>\quad$ Hikayatin ang mga mag-aaral na makibahagi sa panglipunan programa o community outreach na may adhikain pangkomunikasyon, upang magkaroon ng karanasan sa pagbabahagi ng ideya. Magkaroon ng patimpalak ang bawat komunidad, paaralan ukol sa pakikipagtalastasan, bukas sa lahat ng lebel ng edukasyon upang sanayin at mapaunlad ang tiwala sa sarili.

$>\quad$ Ang tagapamanihala ng paaralan, tagapamuno ng komunidad ay kiinakailangan magbigay ng suporta sa programa pangkomunikatibo upang mapataas ang kompyansa sa sarili. Magkaroon ng pagsulat na gawain sa paglinang ng kasanayang komunikatibo ng mga mag-aaral sa iba't ibang lebel ng edukasyon upang mabigyan ng sapat na aksyon lalo na sa panahon ng pandemya at upang mabigyan ng sapat o angkop na programa o gawain na nakalaan sa kanilang lebel.

$>\quad$ Makakatulong ang lokal na pamahalaan sa pagpapaunawa kung gaano kalaki ang gampanin at epekto ng pagpili ng komunikasyon na gagamitin sa pagpapalagan ng mga impormasyon. Mahihikayat ang organisasyon pangkabataan na maging bukas sa palihan o training makakatulong sa pag-unald ng sarili hindi lamang sa kasanayan komunikatibo bagkus sa iba’t ibang kasanayan.

\section{Sanggunian}

Bonabon, M. (2020). Mga danas at dalumat sa paghuhubad sa panahon ng krisis. National Book Development Board.

Dinglasan, R. (2012). Komunikasyon sa akademikong Filipino (Filipino 1). Unang Edisyon. Rex Bookstore. Manila Philippines.

Fortich, C., et al. (2021). Gampanin ng wikang Filipino sa pagpapalaganap ng impormasyon bilang tugon sa COVID-19 sa Lungsod Quezon. https://philpapers.org/archive/givgnw.pdf

Hilario, R., \& Casal, C. (n.d.). Ang komunikasyon bilang instrumento ng wika. Course Hero. https://www.coursehero.com/file/51384750/Komunikasyon-Bilang-Instrumento-ngWikadocx/

Johnson, L. F., et al. (2010). Key emerging technologies for elementary and secondary education. 2010 Horizon Report: K-12 Edition. Retrieve from https://www.eddigest.com

Language as Medium of Communication. (n.d.). Bartly Research. Retrieved from https://www.bartleby.com/essay/LanguageAs-A-Medium-Of-CommunicationPKVHX93TU5YQ

Mandado, J. O. (2020). Ang penomenong awiting visayan popular o (VisPop) sa Kabatang Sebwano at ilang usaping wika at kultura. American Journal of humanities and social sciences research, 4(11), 188-195.

Patanao, F., et al. (1996). Bitaw: Sining ng komunikasyon. Trinity College, Wesleyan University.

Sauco, C. et al. (1978). Pandalubhasaan sining ng komunikasyon. Katha Publishing Co.

Saul, A. J. G., et al. (2020). Manipestasyon ng Ika-21 siglong kasanayan at mungkahing gawaing makalilinang ng kasanayan sa kurikulum ng Filipino ng Ikasampung Baitang. Asia Pacific Higher Education Research Journal 7(1), 41-45.

Varona, F. A., \& Mandado, J. O. (2020). Ang kasanayang pasalita gamit ang kagamitang multimidya sa antas tersyarya. https://www.ajhssr.com/wp-content/uploads/2020/12/y20412180186.pdf 
Especial, M. A., Melitante, M. A., \& Janer, S.

106 Consortia Academia Publishing (A partner of Network of Professional Researchers and Educators) 